\title{
Fisher Syndrome
}

National Cancer Institute

\section{Source}

National Cancer Institute. Fisher Syndrome. NCI Thesaurus. Code C116958.

An autoimmune process characterized by the clinical triad of ophthalmoplegia, ataxia, and areflexia. 